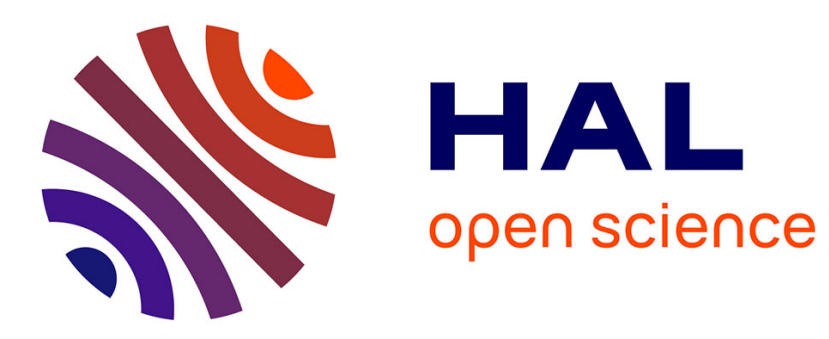

\title{
Prévoir sans persistance
}

Christophe Boucher, Bertrand Maillet

\section{- To cite this version:}

Christophe Boucher, Bertrand Maillet. Prévoir sans persistance. 2012. halshs-00662771

\section{HAL Id: halshs-00662771 https://shs.hal.science/halshs-00662771}

Submitted on 25 Jan 2012

HAL is a multi-disciplinary open access archive for the deposit and dissemination of scientific research documents, whether they are published or not. The documents may come from teaching and research institutions in France or abroad, or from public or private research centers.
L'archive ouverte pluridisciplinaire $\mathbf{H A L}$, est destinée au dépôt et à la diffusion de documents scientifiques de niveau recherche, publiés ou non, émanant des établissements d'enseignement et de recherche français ou étrangers, des laboratoires publics ou privés. 


\section{Documents de Travail du Centre d'Economie de la Sorbonne}

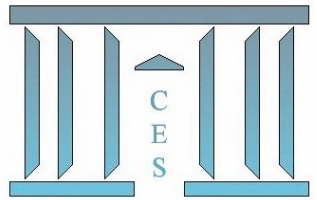

Prévoir sans persistance

Christophe BoucheR, Bertrand MAILlET

2012.01

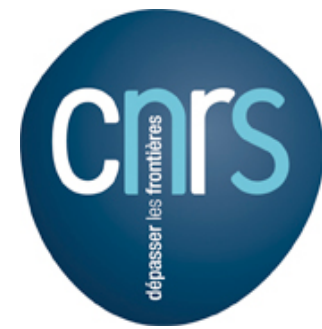


La littérature sur la prévision économique et financière a identifié trois problèmes importants: l'instabilité des régressions prédictives, la discordance des résultats des tests de prévisions en échantillon et hors échantillon, et la difficile inférence statistique lorsque les prédicteurs sont hautement persistants. Dans cet article, nous abordons ces trois questions simultanément, en proposant de traiter en amont la persistance des variables prédictives. Nous retirons ainsi préalablement les composantes basses fréquences des prédicteurs et nous montrons, en simulations et sur des données financières, que ce pré-traitement permet d'améliorer leur pouvoir prédictif.

\section{FORECASTING WITHOUT PERSISTENCE}

The forecasting literature has identified three important and broad issues: the predictive content is unstable over time, in-sample and out-of-sample discordant results and the problematic statistical inference with highly persistent predictors. In this paper, we simultaneously address these three issues, proposing to directly treat the persistence of forecasting variables before use. We thus cut-out the low frequency components and show, in simulations and on financial data, that this pre-treatment improves the predictive power of the studied economic variables.

Classification JEL: C14, C53, G17.

A.A.Advisors-QCG (ABN AMRO), Variances et Université Paris-1 (CES/CNRS). Correspondance à : Christophe Boucher, CES/CNRS, MSE, 106-112 Bd de l'Hôpital F-75647 Paris Cedex 13. Tel. : 0144078189/70 (fax). Courriel : christophe.boucher@univ-paris1.fr.

A.A.Advisors-QCG (ABN AMRO), Variances et Université d'Orléans (LEO/CNRS et IEF). Courriel : bertrand.maillet@univ-orleans.fr.

Nous remercions Olivier Darné, Laurent Ferrara, Christophe Hurlin, Julien Idier, Marie Lambert, Jean-Stéphane Mésonnier, Christophe Pérignon, Sessi Tokpavi et Fabien Tripier pour leurs commentaires, Gregory Jannin pour son assistance, ainsi que les participants des conférences de l'AFFI (Montpellier, mai 2011) et de l'AFSE (Nanterre, septembre 2011) pour leurs suggestions lors de la préparation de ce travail. Le premier auteur remercie la Fondation Banque de France et le second l'Institut Europlace de Finance pour leurs soutiens financiers. L'avertissement habituel s'applique ici. 


\section{INTRODUCTION}

Cet article s'intéresse à trois problèmes importants et clairement identifiés dans la littérature sur la prévision économique et financière. Le premier concerne l'instabilité des régressions prédictives et plus généralement des tests de causalité de Granger. Le deuxième se rapporte à la discordance des résultats des tests de prévision en échantillon et hors échantillon (insample versus out-of-sample). Le troisième a trait à la difficile inférence statistique en présence de prédicteurs hautement persistants. Ces questions ont été discutées, entre autres, par Stock et Watson [2003], Goyal et Welch [2003], Paye et Timmermann [2006], et, Lettau et van Nieuwerburgh [2008].

Nous abordons ces trois questions simultanément dans cet article, en proposant de traiter en amont la persistance des variables prédictives. A la suite de Boucher et Maillet [2011], nous retirons ainsi les composantes basses fréquences des prédicteurs et nous montrons en simulations et sur des données financières que ce pré-traitement permet d'améliorer leur pouvoir prédictif. Notre stratégie de prévision consiste ainsi à utiliser des régressions linéaires standard après avoir détruit la persistance des variables prédictives.

Classiquement, les prévisions économiques et financières sont réalisées à partir de régressions faisant intervenir des variables retardées, en supposant, pour que l'inférence soit valide, que celles-ci sont stationnaires. Dans le domaine financier par exemple, les intervalles de confiance calculés sur les racines autorégressives des principales variables prédictives des rentabilités boursières (telles que le rendement en dividende, le ratio valeur comptable sur valeur boursière, le taux d'intérêt de court-terme ou certains différentiels de taux) confirment l'incertitude quant à l'ordre d'intégration de ces séries (e.g. Torous et al. [2004]). Stambaugh [1999] montre par ailleurs qu'une persistance avérée conduit à des coefficients de régressions prédictives biaisés quand les termes d'innovations sont corrélés avec la variable dépendante. De même, les statistiques standard des $t$ de Student pour ce type de régressions prédictives ne sont pas de niveau correct (Cavanagh et al. [1995]). Ces problèmes sont de plus exacerbés si les études sont réalisées par exploration des données (ou data mining - Ferson et al. [2003]). Enfin, Lettau et van Nieuwerburgh [2008] montrent que la faible performance hors échantillon des prévisions financières est directement liée à l'instabilité des régressions prédictives.

Plusieurs travaux récents, essentiellement appliqués à la prévision financière, se sont intéressés à des moyens de corriger l'inférence approximative entraînée par les propriétés des variables hautement persistantes (Torous et al. [2004] ; Campbell et Yogo [2006] ; Jansson et Moreira [2006] ; Ang et Bekaert [2007]). Ces auteurs concluent de manière générale que les tests de prévisibilité des rentabilités boursières sont moins favorables une fois les corrections apportées. A la suite de Boucher et Maillet [2011] ${ }^{1}$, nous choisissons d'emprunter une autre voie, en «blanchissant » les séries explicatives, i.e. en détruisant préalablement leur persistance, avant de les

\footnotetext{
${ }^{1}$ Dans un précédent article compagnon, Boucher and Maillet [2011] étudient spécifiquement le rôle de différentes composantes du Price Earning Ratio - tirées d'une décomposition par ondelettes, et mesurées ainsi à plusieurs échelles de temps, dans la détermination des rentabilités boursières.
} 
utiliser dans un cadre traditionnel. En éliminant les composantes basses fréquences des prédicteurs, nous traitons par-là même les ruptures structurelles qui affectent leur comportement, ce qui permet d'obtenir des régressions prédictives plus stables dans le temps. Ainsi, notre propos n'est pas ici de s'intéresser uniquement à l'inférence directe d'une estimation, mais d'étudier, dans une stratégie d'estimation à deux étapes (blanchiment puis prévision), le pouvoir prédictif de variables dont les processus sont proches de ceux caractérisés par une racine unitaire. Il s'agit ainsi de rendre les prédicteurs conformes aux hypothèses traditionnelles, pour en étudier ensuite leur pouvoir prédictif final. Nous montrons que ce pré-traitement en amont permet d'obtenir de meilleures prévisions.

D'un point de vue économique, éliminer les composantes basses fréquences permet de se focaliser sur les mouvements de court-terme plus conjoncturels, et de faire abstraction des ruptures structurelles. Ainsi, à des horizons de prévisions relativement courts (quelques mois), les effets de nouvelles informations micro- et macro-économiques arrivant sur les marchés ne sont plus perturbés par les tendances de long-terme perceptibles dans les composantes basses fréquences des séries temporelles.

Le reste de l'article est organisé comme suit. La section 2 présente notre méthodologie et l'illustre à partir de simulations de Monte Carlo. La section 3 évalue le pouvoir prédictif hors échantillon de notre stratégie de prévisions sur des rentabilités boursières aux Etats-Unis. La section 4 conclut.

\section{METHODOLOGIE DE PREVISION ET ILLUSTRATIONS EN SIMULATIONS}

L'identification des prédicteurs économiques et financiers est généralement réalisée à partir de tests de causalité de Granger en échantillon, et le pouvoir prédictif est mesuré alors par le coefficient de détermination de la régression prédictive. Les tests de causalité de Granger consistent à évaluer la significativité du prédicteur potentiel dans une régression de la variable expliquée, $y_{t+h}$, sur le prédicteur retardé, $x_{t}$, où $h$ est l'horizon de prévision, telle que (pour $t=[1, \ldots, T]$ ):

$$
y_{t+h}=\beta_{h}^{\prime} x_{t}+\gamma_{h}^{\prime} z_{t}+\varepsilon_{t+h}
$$

avec $z_{t}$ des variables de contrôle (telles qu'une constante et des retards de la variable $\left.y_{t}\right), \varepsilon_{t+h}$ l'erreur de prévision, et, $\beta_{h}$ et $\gamma_{h}$ les paramètres à estimer.

Notre stratégie de prévision consiste à retirer les composantes basses fréquences du prédicteur potentiel, en utilisant un filtre permettant d'isoler celles-ci. De nombreuses techniques de décomposition et de filtre existent 
dans la littérature. Dans la suite de l'article, nous en considérons trois principales: le filtre de Hodrick-Prescott (HP), une décomposition par ondelettes et une moyenne mobile passée (ou backward).

Graphique 1. Pouvoir prédictif du taux des Bons du Trésor américains sur les rentabilités excédentaires après pré-traitement de la persistance (avec moyenne mobile passée)

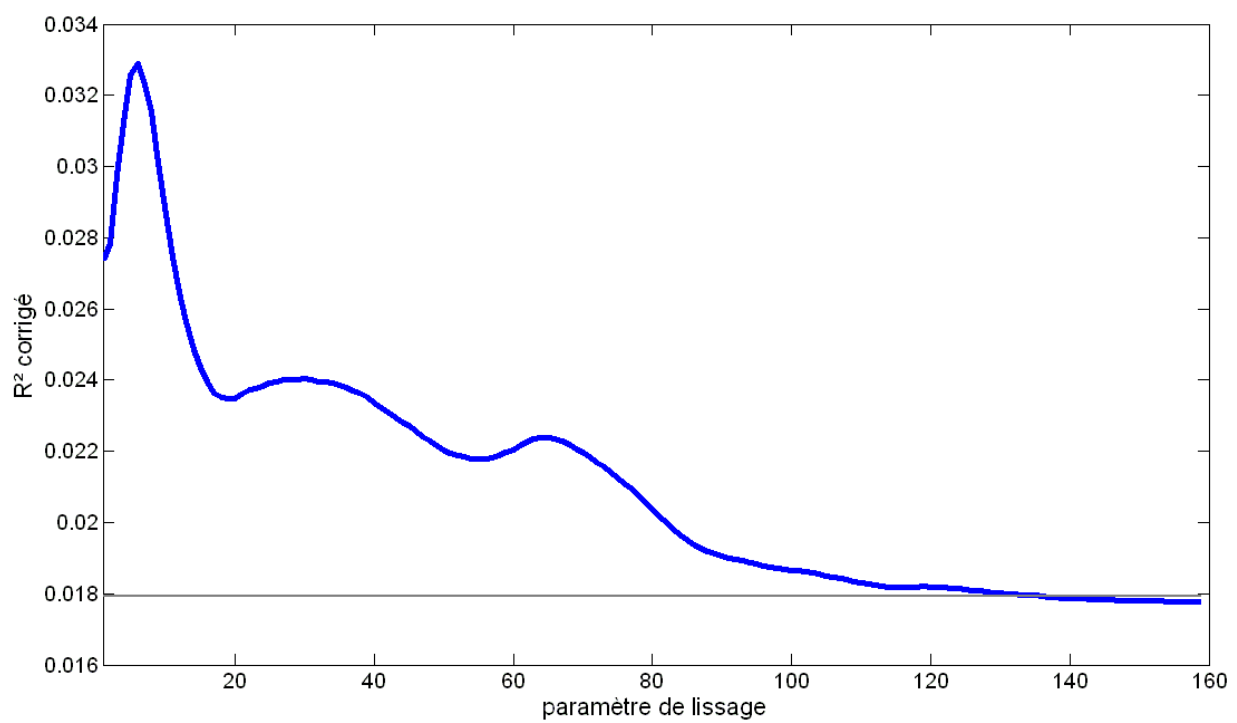

Source: S\&P 500 Earnings and Estimate Report et IPC - données trimestrielles sur la période T4:1934T2:2010; calculs des auteurs. Ce graphique illustre le pouvoir prédictif à un trimestre du taux des Treasury-bills américains sur les rentabilités excédentaires des actions $\left(\mathrm{R}^{2}\right.$ corrigé sur l'axe des ordonnés). La courbe épaisse représente le coefficient de détermination corrigé de la régression prédictive avec comme prédicteur le taux d'intérêt pré-traité (avec une constante et un terme AR(1)), en fonction de la taille de la fenêtre de la moyenne mobile passée (i.e. le paramètre de lissage en nombre de trimestres sur l'axe des abscisses) considérée pour ce taux d'intérêt. Le pré-traitement consiste à soustraire du taux d'intérêt la composante basse fréquence identifiée, avant de procéder à la régression. La ligne fine grise représente le coefficient de détermination corrigé (un seul $\mathrm{R}^{2}$ ) correspondant à une analyse menée avec la série des taux des Treasury-bills sans pré-traitement.

Le filtre de Hodrick-Prescott [1997] est probablement une des méthodes les plus usitées en macroéconomie pour identifier et séparer les mouvements de long-terme des fluctuations cycliques dans une série temporelle. Toutefois, son utilisation lors d'exercices de prévision hors échantillon pourra être limitée compte tenu de sa décomposition «tournée vers l'avant» (ou forward). L'analyse par ondelettes est une alternative aux filtres passe-bandes ${ }^{1}$ car elle offre une meilleure résolution dans le domaine du temps (Wang [1995]). De fait, les fonctions de bases (ondelettes) sont localisées à la fois dans les fréquences mais aussi dans le temps ${ }^{2}$, ce qui nous est ici utile pour capturer la datation et la magnitude de changements persistants des prédicteurs. Le filtre par moyenne mobile passée est ici considéré comme une méthode simple de référence.

\footnotetext{
${ }^{1}$ Un filtre passe-bande est un filtre ne laissant passer qu'une bande ou intervalle de fréquences compris entre une fréquence de coupure basse et une fréquence de coupure haute du filtre.

${ }^{2}$ L'analyse par ondelettes consiste en une décomposition d'un signal en un ensemble de fonctions de base (les ondelettes) de façon analogue aux fonctions sinus et cosinus utilisées dans la transformée de Fourier. Ces ondelettes sont obtenues par translation et dilatation-contraction d'une ondelette mère. Toutefois, dans l'analyse de Fourier, toute notion de localisation temporelle disparait dans l'espace de Fourier. Les ondelettes renseignent au contraire sur le contenu fréquentiel tout en préservant la localisation afin d'obtenir une représentation temps/fréquence.
} 
Le Graphique 1 illustre notre intuition. En appliquant une méthode basique - telle qu'une moyenne mobile passée - sur le taux d'intérêt de courtterme pour prévoir les rentabilités boursières excédentaires aux Etats-Unis ${ }^{1}$, le pouvoir prédictif est amélioré une fois que les composantes basses fréquences sont retirées. Ce graphique montre que le coefficient de détermination corrigé (axe des ordonnés) de la régression prédictive trimestrielle, évolue en fonction de la fenêtre de lissage (axe des abscisses) retenue pour retirer les basses fréquences du taux d'intérêt. Le pouvoir prédictif (mesuré par le $R^{2}$ ) est une fonction non-linéaire de la taille de la fenêtre de calcul de la moyenne mobile qui est retirée au taux d'intérêt. Cette relation est d'abord croissante, pour atteindre un pic correspondant à une destruction de la moyenne mobile à horizon de 6 mois, pour décroître ensuite et tendre vers le niveau (faible) obtenu avec la série temporelle originelle du taux d'intérêt.

Soit le système tel que :

$$
\left\{\begin{array}{l}
y_{t}=\phi_{1} y_{t-1}+\zeta_{t+1} \\
x_{t}=\phi_{2} x_{t-1}+\kappa y_{t+1}+\xi_{t+1}
\end{array}\right.
$$

avec $\zeta_{t+1}$ et $\xi_{t+1}$, deux bruits blancs Gaussiens.

Nous simulons maintenant ce système à partir de 10000 tirages d'échantillons de taille modeste (de $T=1000$ observations pour chaque simulation), en considérant que la variable expliquée $\left(y_{t}\right)$ est causée (avec $\kappa=1$ ) ou non (i.e. $\kappa=0)$ par le prédicteur $\left(x_{t}\right)$, et ceci pour différents degrés de persistance de la variable dépendante et du prédicteur (respectivement $\phi_{1}$ et $\phi_{2}$ ).

Nous estimons ensuite une régression prédictive équivalente à l'équation (1) avec une constante, mais sans terme autorégressif, de façon à identifier directement le pouvoir prédictif mesuré par le coefficient de détermination corrigé, indépendamment de la mémoire du processus suivi par la variable dépendante $^{2}$. Les Graphiques 2 et 3 présentent, pour les trois méthodes avec pré-traitement visant à supprimer les composantes basses fréquences (Hodrick-Prescott, moyenne mobile et ondelettes) et celle sans pré-traitement, le pouvoir prédictif de $x_{t}$ selon différentes combinaisons des paramètres du système (2). Pour chacune des méthodes de lissage, nous avons retenu les paramètres visant à extraire le maximum de basses fréquences (extraction de la composante de la plus haute fréquence pour chacune des méthodes) ${ }^{3}$. Dans tous les cas, le prédicteur est considéré comme fortement persistant mais stationnaire $\left(\phi_{2}=0,98\right)$. Dans un premier temps, nous considérons dans les simulations suivantes que $x_{t}$ ne contient aucune information quant à la

\footnotetext{
${ }^{1}$ La base de données est décrite dans la section suivante.

${ }^{2}$ Les résultats (disponibles auprès des auteurs) restent qualitativement les mêmes en considérant un terme autorégressif dans la régression prédictive.

${ }^{3} \mathrm{Ce}$ paramètre de lissage n'est cependant pas forcément celui qui permet d'extraire le plus d'information prédictive à court-terme, comme le montre le Graphique 1 où le pouvoir prédictif augmente au départ avec le paramètre de lissage.
} 
réalisation de $y_{t+1}($ i.e. $\kappa=0)$ en distinguant d'abord le cas où la variable expliquée n'est pas persistante $\left(\phi_{1}=0\right)$, puis le cas où elle l'est $\left(\phi_{1}=0,98\right)$.

Graphique 2. Pouvoir prédictif mesuré en l'absence de contenu informationnel du prédicteur

\section{Paramètres :}

$\phi_{1}=0 ; \phi_{2}=0,98 ; \kappa=0$
Paramètres :

$$
\phi_{1}=0,98 ; \phi_{2}=0,98 ; \kappa=0
$$
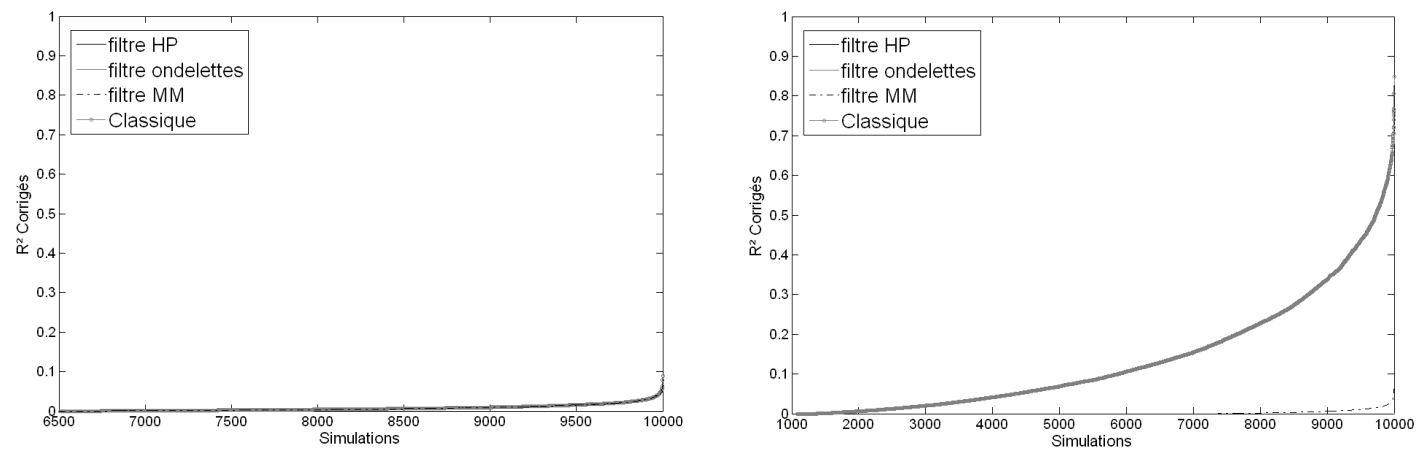

Source : simulations ; calculs des auteurs. Ces graphiques représentent en ordonné le pouvoir prédictif, mesuré par le coefficient de détermination corrigé (classé en ordre croissant) de la régression prédictive avec comme prédicteur, $x_{t}$, (pré-traité ou non), avec comme variable dépendante, $y_{t}$. Nous avons considérés 10000 tirages des séries $x_{t}$ et $y_{t}($ avec $T=200)$. Leurs processus sont définis par le système (2).

Le Graphique 2 montre que le risque de régression prédictive fallacieuse n'est pas négligeable lorsque la variable dépendante et le prédicteur sont fortement persistants (mais stationnaires, avec $\phi_{1}=\phi_{2}=0,98$ ) puisque respectivement $65 \%$ et $45 \%$ des régressions sans pré-traitement (régression dite «classique ») présentent un $R^{2}$ supérieur à $5 \%$ et $10 \%$ (et $25 \%$ des $t$ statistiques sont supérieures à 3 en valeur absolue $)^{1}$. Par contraste, le prétraitement par suppression des composantes basses fréquences réduit drastiquement le risque de seconde espèce (probabilité de $2 \%$ de $t$-statistiques supérieures à 3 en valeur absolue).

Le Graphique 3 présente les résultats des régressions prédictives lorsque $x_{t}$ possède maintenant un pouvoir prédictif sur $y_{t+1}($ avec $\kappa=1)$. Nous distinguons deux cas : l'absence de persistance dans le processus suivi par la variable dépendante $\left(\phi_{1}=0\right)$ et une faible persistance $\left(\phi_{1}=0,1\right)$. Il apparaît que le filtre Hodrick-Prescott, suivi par le filtre par ondelettes et par celui de la moyenne mobile, est le plus efficace pour extraire la composante contenant l'information prédictive en échantillon pour les deux niveaux de persistance considérés. En revanche, le pouvoir prédictif de la variable non traitée (régression « classique ») apparaît beaucoup plus faible avec un $R^{2}$ inférieur à $10 \%$ dans $65 \%$ des simulations ${ }^{2}$.

Nous examinons dans la section suivante les propriétés (précision et stabilité) hors échantillon de notre méthodologie de prévision sur des données

\footnotetext{
${ }^{1}$ Les valeurs des statistiques de Student correspondantes sont disponibles auprès des auteurs.

${ }^{2}$ En revanche, lorsque la variable dépendante est fortement persistante $\left(\phi_{1}=0,98\right)$, le pré-traitement ne permet plus de capturer le pouvoir prédictif contenu dans $x_{t}$. Les résultats de ces simulations sont disponibles auprès des auteurs.
} 
financières. En effet, les exercices de prévision en échantillon sont sujets au biais de connaissance a priori de l'échantillon total.

Graphique 3. Pouvoir prédictif mesuré avec contenu informationnel du prédicteur

Paramètres :

$\phi_{1}=0 ; \phi_{2}=0,98 ; \kappa=1$

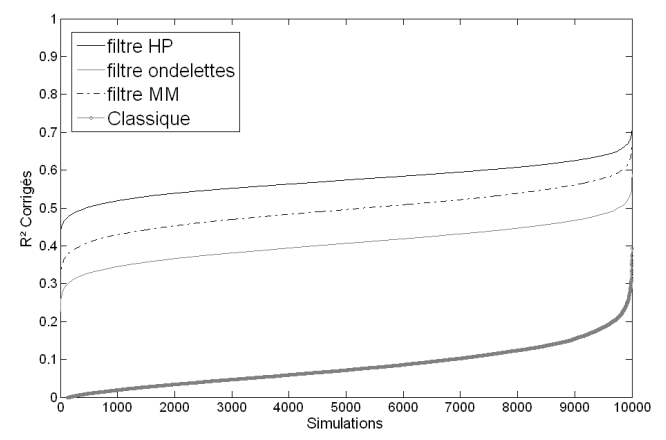

Paramètres :

$\phi_{1}=0,1 ; \phi_{2}=0,98 ; \kappa=1$

Source: simulations; calculs des auteurs. Ces graphiques représentent en ordonné le pouvoir prédictif, mesuré par le coefficient de détermination corrigé (classé en ordre croissant) de la régression prédictive avec comme prédicteur, $x_{t}$, (pré-traité ou non), avec comme variable dépendante, $y_{t}$. Nous avons considérés 10000 tirages des séries $x_{t}$ et $y_{t}$ (avec $T=200$ ). Leurs processus sont définis par le système (2).

\section{PREVISIONS BOURSIERES SANS PERSISTANCE HORS- ECHANTILLON}

Notre étude empirique porte sur des données américaines trimestrielles qui couvrent la période T4:1934-T2:2010. Le logarithme des cours, $p_{t}$, et des dividendes, $d_{t}$, du Standard \& Poor's $(S \& P)$ sont tirés de Campbell et Shiller [1988] et ils sont actualisés à partir du site internet du $S \& P$ ( $S \& P 500$ Earnings and Estimate Report). Les autres données macro-économiques et financières sont tirées de la base de données FRED II de la Réserve Fédérale de Saint Louis. Nous avons retenu trois prédicteurs, parmi les plus mobilisés dans les études empiriques, des rentabilités excédentaires des actions (i.e. les primes de risque relativement au taux des Bons du Trésor américain à 3 mois) : le rendement en dividende, $d y_{t}$, le taux d'intérêt des Bons du Trésor à 3 mois, $t b_{t}$, et le spread de défaut, $D E F_{t}$, défini comme la différence entre les taux rémunérant les obligations $\mathrm{BAA}$ et ceux qui correspondent à une notation AAA.

Nous opérons deux types de comparaison pour évaluer le pouvoir prédictif hors échantillon de notre stratégie de prévision : des comparaisons imbriquées et d'autres non-imbriquées (nested comparisons et non-nested comparisons). Pour les comparaisons imbriquées, nous comparons un modèle contraint (avec un terme autorégressif et une constante) avec un modèle noncontraint qui inclut, en plus des mêmes variables que le modèle contraint, une variable supplémentaire potentiellement prédictive. Pour les comparaisons 
non-imbriquées, nous comparons la performance de deux modèles concurrents, c'est-à-dire liés à deux variables prédictives différentes.

Nous utilisons quatre statistiques pour mener ces comparaisons : le ratio des erreurs de prévision quadratiques moyennes (MSE), le test d'encadrement (encompassing test) ENC-NEW de Clark et McCracken [2001], le test d'équivalence des pouvoirs prédictifs MSE-F de McCracken [2004], et le test d'encadrement de Diebold-Mariano modifié (MDM) proposé par Harvey et al. [1998]. Nous appliquons les tests ENC-NEW et MSE-F pour les comparaisons imbriquées et le test MDM pour les comparaisons nonimbriquées. Le ratio des MSE est présenté pour les deux types de comparaison. ${ }^{1}$

Tableau 1. Prévisions de rentabilités excédentaires trimestrielles : comparaisons imbriquées

\begin{tabular}{|c|c|c|c|c|}
\hline \# & $\begin{array}{c}\text { Modèles } \\
\text { non-contraints }\end{array}$ & $M S E_{n d} M S E_{c}$ & $\begin{array}{c}\text { Test } \\
E N C-N E W\end{array}$ & $\begin{array}{c}\text { Test } \\
M S E-F\end{array}$ \\
\hline \multicolumn{5}{|c|}{ Régression $\mathrm{n}^{\circ} 1:$ Rendement en dividende $\left(d y_{t}\right)$} \\
\hline 1 & Sans pré-traitement & 0,9948 & 1,6790 & 0,5074 \\
\hline 2 & Hodrick-Prescott & 1,0086 & 5,5211 & $-0,8360$ \\
\hline 3 & Moyenne Mobile & 0,9653 & $3,0512 * *$ & $3,5198 *$ \\
\hline 4 & Ondelettes & 0,9327 & $6,9503 * *$ & $7,0745 * *$ \\
\hline \multicolumn{5}{|c|}{ Régression $\mathrm{n}^{\circ} 2:$ Bons du Trésor $\left(t b_{t}\right)$} \\
\hline 5 & Sans pré-traitement & 0,9892 & 2,7735 & 1,0689 \\
\hline 6 & Hodrick-Prescott & 0,9667 & $3,5402 *$ & $3,3732 *$ \\
\hline 7 & Moyenne Mobile & 0,9656 & $3,3630 *$ & $3,4907 *$ \\
\hline 8 & Ondelettes & 0,9646 & $3,0982 *$ & $3,5925^{*}$ \\
\hline \multicolumn{5}{|c|}{ Régression $\mathrm{n}^{\circ} 3:$ Spread de défaut $\left(D E F_{t}\right)$} \\
\hline 9 & Sans pré-traitement & 0,9685 & 2,9790 & $3,1831 *$ \\
\hline 10 & Hodrick-Prescott & 1,0127 & 2,9544 & $-1,2315$ \\
\hline 11 & Moyenne Mobile & 0,9672 & $3,1108 *$ & $3,3198 *$ \\
\hline 12 & Ondelettes & 0,9661 & $3,2405^{*}$ & $3,4431 *$ \\
\hline
\end{tabular}

Nous considérons d'abord les deux premiers tiers des observations pour l'estimation initiale en échantillon (de T4:1934 à T1:1984), et, ensuite, nous formons les prévisions hors échantillon de manière récursive pour le reste de l'échantillon total'2.

Le tableau 1 présente les résultats des tests imbriqués des prévisions de rentabilités excédentaires hors échantillon à l'horizon d'un trimestre. Ces résultats montrent que les modèles non-contraints (qui incluent le prédicteur brut ou le prédicteur pré-traité) présentent des erreurs de prévision quadratiques moyennes plus faibles que les modèles contraints, à l'exception

\footnotetext{
${ }^{1}$ De manière générale, ce ratio permet de vérifier si le modèle au numérateur du ratio présente des erreurs de prévisions plus faibles (ratio inférieur à un) que le modèle au dénominateur. Les différents tests permettent de vérifier que ces différences sont significatives.

${ }^{2}$ En utilisant les paramètres de lissage pour les 3 filtres qui présentaient le pouvoir prédictif (mesuré par le $R^{2}$ corrigé) le plus élevé lors de l'estimation initiale.
} 
du pré-traitement par le filtre Hodrick-Prescott. Par ailleurs, les deux tests ENC-NEW et MSE-F rejettent l'hypothèse nulle que le pré-traitement par ondelettes et par moyenne mobile conduisent à un pouvoir prédictif nonsignificatif au seuil de 5\%. Enfin, les erreurs moyennes de prévision des modèles avec le pré-traitement par ondelettes apparaissent toujours inférieures à celles correspondant aux autres méthodes de filtrage.

Examinons maintenant si ces différences de pouvoir prédictif entre les méthodes elles-mêmes sont significatives. Le tableau 2 présente les résultats du test non-imbriqué de prévision MDM. Les prévisions à l'aide du prétraitement par ondelettes apparaissent toujours de meilleure qualité (ratio des MSE). Mais ces prévisions n'apparaissent pas significativement différentes au seuil de $5 \%$ de celles obtenues à partir des autres méthodes de filtrage. ${ }^{1}$

Tableau 2. Prévisions de rentabilités excédentaires trimestrielles: comparaisons non-imbriquées

\begin{tabular}{|c|c|c|c|c|}
\hline $\mathrm{N}^{\circ}$ & Modèle 1 versus Modèle 2 & $M S E_{1} / M S E_{2}$ & Test MDM & p-statistique \\
\hline \multicolumn{5}{|c|}{ Régression $\mathrm{n}^{\circ} 1:$ Rendement en dividende $\left(d y_{t}\right)$} \\
\hline 1 & Ondelettes vs. Hodrick-Prescott & 0,9247 & 1,3090 & 0,1936 \\
\hline 2 & Ondelettes vs. Moyenne Mobile & 0,9662 & 0,8495 & 0,3977 \\
\hline \multicolumn{5}{|c|}{ Régression $\mathrm{n}^{\circ} 2$ : Bons du Trésor $\left(t b_{t}\right)$} \\
\hline 3 & Ondelettes $v s$. Hodrick-Prescott & 0,9978 & 0,0850 & 0,9325 \\
\hline 4 & Ondelettes vs. Moyenne Mobile & 0,9990 & 0,0602 & 0,9522 \\
\hline \multicolumn{5}{|c|}{ Régression $n^{\circ} 3:$ Spread de défaut $\left(D E F_{t}\right)$} \\
\hline 5 & Ondelettes $v s$. Hodrick-Prescott & 0,9539 & 1,4232 & 0,1579 \\
\hline 6 & Ondelettes vs. Moyenne Mobile & 0,9988 & 0,1041 & 0,9173 \\
\hline \multicolumn{5}{|c|}{$\begin{array}{l}\text { Source: S\&P } 500 \text { Earnings and Estimate Report et IPC-données trimestrielles sur la période T4:1934- } \\
\text { T2:2010 ; calculs des auteurs. Toutes les régressions incluent une constante. L'hypothèse nulle est que le } \\
\text { Modèle } 2 \text { n'apporte pas d'information supplémentaire relativement au Modèle 1. La colonne "MSE1/MSE2" } \\
\text { présente les erreurs de prévisions quadratiques moyennes du Modèle } 1 \text { relativement à celles du Modèle 2. }\end{array}$} \\
\hline
\end{tabular}

\section{CONCLUSION}

Dans cet article, nous proposons de traiter en amont la persistance des prédicteurs. Nous montrons, en simulations et sur des données financières, qu'éliminer ex ante une partie de la persistance des prédicteurs permet d'améliorer sensiblement leur pouvoir prédictif.

Notre stratégie en deux étapes (blanchiment puis prévision) est donc une alternative pratique aux méthodes visant à optimiser l'inférence statistique lorsque le prédicteur est persistant, mais également aux stratégies de prévisions avec rupture telles que les régressions en moyenne sur différentes fenêtres temporelles (e.g. Pesaran et Timmermann [2007]).

Il conviendra d'examiner sur des données macroéconomiques, l'efficacité de notre stratégie de prévisions en deux étapes. D'autres critères (e.g. en termes d' « équivalent certain ») et d'autres tests pourront également

\footnotetext{
${ }^{1}$ Le principe du pré-traitement est donc ainsi plus important que la méthode utilisée pour l'appliquer.
} 
être implémentés pour assurer la robustesse de nos résultats. Enfin, il pourrait être utile de comparer les résultats de notre approche en deux étapes avec les méthodes directes d'inférence avec prédicteur persistant.

\section{RÉFÉRENCES BIBLIOGRAPHIQUES}

Ang A. et BeKaert G. [2007], « Stock Return Predictability: Is it There? », Review of Financial Studies, 20(3), 651-707.

Boucher C. et MaILlet B. [2011], «Une analyse multi-fréquences des cycles financiers », Revue Economique, 62(3), 441-450.

CAMPBell J.Y. et ShILleR R.J. [1988], « Stock Prices, Earnings and Expected Dividends », Journal of Finance, 43(3), 661-676.

CAmpbell J.Y. et Yogo M. [2006], «Efficient Tests of Stock Return Predictability », Journal of Financial Economics, 81(1), 27-60.

Cavanagh C.L., Elliot G. et Stock J.H. [1995], « Inference in Models with Nearly Integrated Regressors », Econometric Theory, 11(5), 1131-1147.

Clark T.E. et MCCRAKEN M.W. [2001], "Tests of Equal Forecast Accuracy and Encompassing for Nested Models », Journal of Econometrics, 105(1), 85-110.

FERSON W.E., SARKISSIAN S. et Simin T.T. [2003], "Spurious Regressions in Financial Economics? », Journal of Finance, 58(4), 1393-1413.

Harvey D., Leybourne S. et Newbold P. [1997], "Testing the Equality of Prediction Mean Squared Errors », International Journal of Forecasting, 13(2), 281-291.

Hodrick R.J. et PrescotT E.P. [1997], «Post-war Business Cycles An Empirical Investigation », Journal of Money, Credit and Banking, 29(1), 1-16.

JANSSON M. et MOREIRA M.J. [2006], «Optimal Inference in Regression Models with Nearly Integrated Regressors », Econometrica, 74(3), 681-715.

LETTAU M. et VAN NIEUWERBURGH S. [2008], « Reconciling the Return Predictability Evidence », Review of Financial Studies 21(4), 1607-1652.

MCCRAKEN M.W. [2007], "Asymptotics for Out-of-sample Tests of Granger Causality », Journal of Econometrics, 140(2), 719-752.

Nelson C.R. et KIM M.J. [1993], « Predictable Stock Returns: the Role of Small Sample Bias », Journal of Finance, 48(2), 641-661.

Pesaran M.H. et TimmermanN A. [2007], « Selection of Estimation Window in the Presence of Breaks », Journal of Econometrics, 137(1), 134-161.

StAmbaugh R.F. [1999], " Predictive Regressions », Journal of Financial Economics, 54(3), 375-421.

Torous W., Valkanov R. et Yan S. [2004], « On Predicting Stock Returns with Nearly Integrated Explanatory Variables », Journal of Business, 77(4), 937966.

WANG Y. [1995], «Jump and Sharp Cusp Detection by Wavelets », Biometrika, 82(2), 385-397. 